\title{
Catalytic Asymmetric Cyclopropanation with Diazooxindole
}

\author{
Atsuko Awata, Takayoshi Arai* \\ Department of Chemistry, Graduate School of Science, Chiba University, Inage, Chiba 263-8522, Japan \\ Fax +81(43)2902889; E-mail: tarai@faculty.chiba-u.jp \\ Received: 12.10.2012; Accepted after revision: 12.11.2012
}

\begin{abstract}
The first catalytic asymmetric cyclopropanation using styrene and diazooxindole was achieved with $\mathrm{Rh}_{2}(S \text {-PTTL })_{4}$. The reaction proceeded smoothly with $1 \mathrm{~mol} \%$ catalyst loading to provide a good yield of the biologically important spiro-cyclopropyloxindole product with moderate to good enantioselectivity and excellent diastereoselectivity.
\end{abstract}

Key words: asymmetric catalysis, spiro compounds, carbenoids, cyclopropanation, oxindole

Spirooxindole is a privileged heterocyclic motif that exists in a large number of bioactive natural alkaloids and pharmaceutical candidates. ${ }^{1-3}$ For example, MI-219 is an inhibitor of the p53-MDM2 protein-protein interaction (Figure 1). ${ }^{4}$ A highly functionalized spiro cyclobutyloxindole, welwitindolinone A isonitrile isolated from bluegreen algae by Moore et al. possesses antifungal activity. ${ }^{5,6}$ Recently, spirooxindoles having spiro[2,4]system have been studied for application to medicinal chemistry. Spiro cyclopropyloxindole 1, especially, is a potent HIV-1 non-nucleoside reverse transcriptase inhibitor. ${ }^{7}$ Spiro epoxyoxindole 2 also shows biological activity as a potent inhibitor of differentiation in promyelocytic leukemia cells. ${ }^{8}$ The biological activity of many organic compounds is closely linked to stereochemistry, a phenomenon referred to as the 'lock-and-key' model. Because of the potent biological importance of these compounds, catalytic enantio- and diastereoselective methods are needed for the synthesis of the spirooxindole framework.

The enantioselective synthesis of these scaffolds is difficult because they have a highly hindered spirocyclic quaternary carbon center. Specifically, the catalytic chiral asymmetric synthesis of spirooxindole containing a cyclopropane ring remains a challenging task in the current organic synthesis. ${ }^{9,10}$ In a program for the development of biologically significant compounds, the stereoselective construction of spirooxindoles has also been of our interest. ${ }^{11}$ Recently, Baltoli and Bencivenni et al. reported the first enantioselective access to spiro cyclopropyloxindoles via an organocatalytic Michael-alkylation cascade reaction using methyleneindolinone and bromonitromethane to give the nitrofunctionalized spiro cyclopropyloxindoles. $^{12}$ For accessing the spiro cyclopropyloxindoles, metal-catalyzed cyclopropanation using diazo compounds of alkenes has provided an alternative approach.

SYNLETT 2013, 24, 0029-0032

Advanced online publication: 11.12.2012

DOI: 10.1055/s-0032-1317746; Art ID: ST-2012-U0880-L

(C) Georg Thieme Verlag Stuttgart · New York
The catalytic asymmetric cyclopropanation using various olefins with diazooxindoles would provide a reliable method for preparing chiral spiro cyclopropyloxindoles containing various functional groups (Scheme 1). Carreira et al. (2003) reported a racemic synthesis of spiro cyclopropyloxindoles through cyclopropanation with diazooxindole for the total synthesis of spirotryprostatin $\mathrm{B} .{ }^{13}$ This report describes a catalytic asymmetric synthesis of spiro cyclopropyloxindoles through transition-metal-catalyzed asymmetric cyclopropanation with diazooxidole.<smiles>CC(C)(C)C[C@H]1N[C@@H](C(=O)NCCC(O)CO)[C@H](c2cccc(Cl)c2)[C@]12C(=O)Nc1cc(Cl)c(F)cc12</smiles>

MI-219<smiles>O=C1Nc2ccc(Cl)cc2C12CC2c1cccs1</smiles>

$$
1
$$

HIV-nonnucleoside reverse transciptase inhibitor

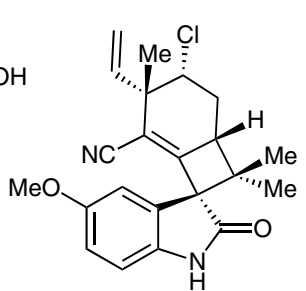

(-)-welwitindolinone A isonitrile

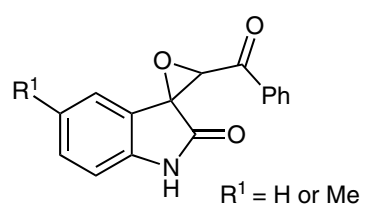

2

antitubercular agents
Figure 1 Biologically active spirooxindole compounds

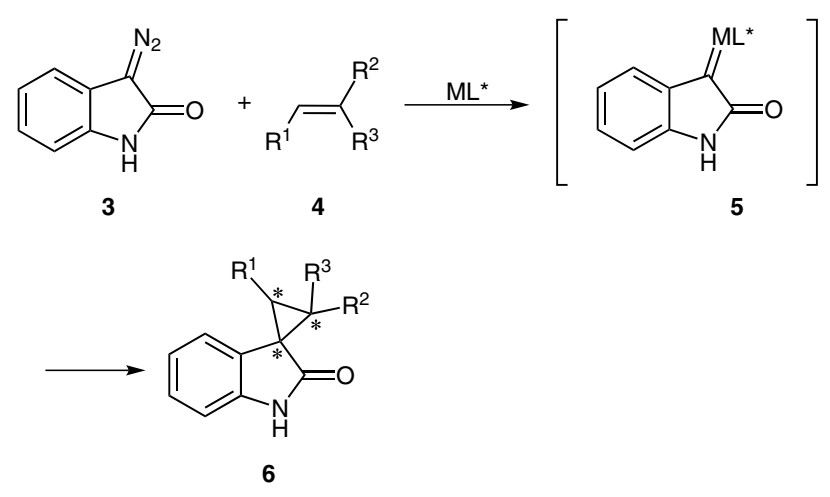

Scheme 1 Catalytic asymmetric synthesis of spiro cyclopropyloxindole via a metal carbenoid intermediate

Racemic cyclopropanation of the diazooxindole and styrene with several metal salts was examined initially (Table 1). A solution of diazooxindole in dichloromethane 
was added slowly to a mixture of styrene, metal salt, and solvent at room temperature. Initially, $\mathrm{Cu}(\mathrm{I})$ triflate, an efficient catalyst for cyclopropanation, was used. ${ }^{14}$ However, the reaction became sluggish, despite consumption of the diazooxindole (Table 1 entry 1 ). The use of the more electron-enriched ethyl vinyl ether also failed to give the desired product. The reaction using $\left[\mathrm{Ru}(p \text {-cymene }) \mathrm{Cl}_{2}\right]_{2}$ resulted in only a trace amount of product (Table 1 entry 2). ${ }^{15}$ In addition, $\mathrm{Cu}(\mathrm{I})$ and $\mathrm{Co}(\mathrm{II})$ acetate did not promote the reaction, and starting material was recovered (Table 1, entries 3 and 4). ${ }^{16}$ In contrast, $\mathrm{Rh}_{2}(\mathrm{OAc})_{4}$ smoothly catalyzed the reaction to give the cylopropanation adduct in 97\% yield with 95:5 diastereoselectivity (Table 1, entry 5). ${ }^{17}$ The NOE analysis of the major product revealed that the amide moiety of oxindole and the phenyl group was in trans configuration (see Supporting Information).

Table 1 Racemic Cyclopropanation of Diazooxindole<smiles>N#CC1C(=O)Nc2ccccc21</smiles><smiles>O=C1Nc2ccccc2[C@]12C[C@H]2c1ccccc1</smiles>

slow addition $(3 \mathrm{~h})$

\begin{tabular}{llll}
\hline Entry & Metal salt & Yield (\%) & trans $/$ cis $^{\text {. }}$ \\
\hline 1 & CuOTf & n.o. & - \\
$2^{\mathrm{a}}$ & {$\left[\operatorname{Ru}(p \text {-cymene }) \mathrm{Cl}_{2}\right]_{2}$} & trace & - \\
3 & $\mathrm{CuOAc}$ & n.r. & - \\
4 & $\mathrm{Co}(\mathrm{OAc})_{2}$ & n.r. & \\
$5^{\mathrm{a}}$ & $\mathrm{Rh}_{2}(\mathrm{OAc})_{4}$ & $>99$ & $95: 5$ \\
\hline
\end{tabular}

${ }^{\text {a }}$ Conditions: $5 \mathrm{~mol} \%$ catalyst were used.

${ }^{\mathrm{b}}$ n.o. $=$ not obtained; n.r. $=$ no reaction.

Initial screening of metal salts resulted in the use of chiral rhodium(II) catalyst, and results summarizing commercially available chiral rhodium catalysts are shown in Table 2. Davies et al. showed that the proline-derived chiral dirhodium carboxylate, $\mathrm{Rh}_{2}(S \text {-TBSP })_{4}$, is an effective catalyst for enantioselective cyclopropenation reactions with aryl diazoacetates. ${ }^{18}$ Although $\mathrm{Rh}_{2}(S \text {-TBSP })_{4}$ smoothly promoted the reaction, product was obtained in only $8 \%$ ee (Table 2 , entry 1$)$. However, $\mathrm{Rh}_{2}(S \text {-PTTL })_{4}$, which was developed by Hashimoto et al., provided enantiomerically enriched spiro cyclopropyloxindole (Table 2, entry 2). ${ }^{19}$ The reaction proceeded in a highly trans-selective manner to give the product in $90 \%$ yield with $61 \%$ ee. Although the reaction at $-40{ }^{\circ} \mathrm{C}$ did not improve the enantioselectivity, the catalyst loading could be reduced to $1 \mathrm{~mol} \%$ (Table 2, entry 5). Furthermore, similar yield and selectivity were obtained by addition of a dichloromethane solution of diazooxindole in one portion (Table 2, entry 6).

The substrate scope of the Rh(II)-catalyzed cyclopropanation was explored, and the results are summarized in Table 3. Styrenes containing an electron-withdrawing group
Table 2 Catalytic Asymmetric Cyclopropanation with Diazooxindole<smiles>N=C1C(=[OH+])Nc2ccccc21</smiles>

slow addition $(3 \mathrm{~h})$

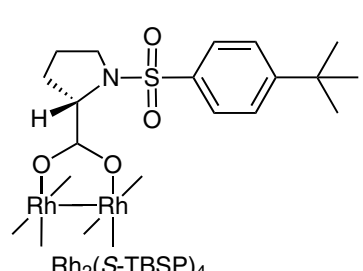

$\mathrm{Rh}_{2}(\mathrm{~S} \text {-TBSP })_{4}$

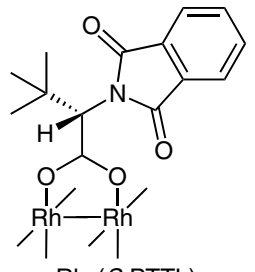

$\mathrm{Rh}_{2}(\mathrm{~S}-\mathrm{PTTL})_{4}$

Entry Catalyst $\quad \mathrm{X} \quad \mathrm{Temp}_{(\mathrm{O})}^{\mathrm{Y} \text { Yield }}$ trans/cis ee of trans

$\left({ }^{\circ} \mathrm{C}\right) \quad(\%)^{\mathrm{a}} \quad(\%)^{\mathrm{b}} \quad(\%)$

\begin{tabular}{lllrrrr}
\hline 1 & $\mathrm{Rh}_{2}(S \text {-TBSP })_{4}$ & 5 & r.t. & 77 & $94: 6$ & 8 \\
2 & $\mathrm{Rh}_{2}(S \text {-PTTL })_{4}$ & 5 & r.t. & 90 & $97: 3$ & 61 \\
3 & $\mathrm{Rh}_{2}(S \text {-PTTL })_{4}$ & 2.5 & 0 & $>99$ & $98: 2$ & 66 \\
4 & $\mathrm{Rh}_{2}(S \text {-PTTL })_{4}$ & 2.5 & -40 & 31 & $97: 3$ & 66 \\
5 & $\mathrm{Rh}_{2}(S \text {-PTTL })_{4}$ & 1 & 0 & $>99$ & $98: 2$ & 66 \\
$6^{c}$ & $\mathrm{Rh}_{2}(S \text {-PTTL })_{4}$ & 1 & 0 & $>99$ & $97: 3$ & 66 \\
\hline
\end{tabular}

${ }^{a}$ Combined yield of diastereomer.

${ }^{\mathrm{b}}$ Determined by crude NMR.

${ }^{\mathrm{c}}$ Diazooxindole was added in one portion and stirred for $1 \mathrm{~h}$.

at the 4-position on the phenyl ring gave the product in moderate enantioselectivity with excellent diastereoselectivity (Table 3, entries 1 and 4). 4-Methylstyrene afforded the product with similar selectivity (Table 3, entry 5 ). The reaction using 4-methoxystyrene was also possible and produced a high chemical yield of product with $48 \%$ ee (Table 3, entry 6). The reaction using 1-pentene gave the product in $65 \%$ yield with acceptable diastereoselectivity (trans $/$ cis $=88: 12$, Table 3, entry 7 ). The trans product exhibited greater enantioselectivity in up to $74 \%$ ee.

The high trans selectivity observed in Tables 1-3 is explained in Scheme 2. For the Rh-catalyzed cyclopropanation using aryl diazoacetate, ${ }^{18 c, 20}$ the styrene attacks with its phenyl group pointing away from the bulky rhodium surface to avoid unfavorable interaction (TS1 in Scheme 2 ). The $\mathrm{C}=\mathrm{C}$ bond of styrene is expected to approach from the amide side because a partial positive charge in the $\alpha$ position of the phenyl group is stabilized by the negative charge of the amide oxygen (TS2). ${ }^{22,23}$ Then, the alkene rotates to form spiro cyclopropyloxindole (TS3). As a result, the trans relation of the amide carbonyl and phenyl group is expected to be the most favorable. The reaction mechanism via the perpendicular approach of the alkene to the rhodium(II)-carbon axis is also possible. ${ }^{19 f, 21}$

In conclusion, the chiral rhodium-catalyzed catalytic asymmetric cyclopropanation of diazooxindole for con- 
Table 3 Catalytic Asymmetric Cyclopropanation of Diazooxindole with Various Olefins ${ }^{\mathrm{a}}$

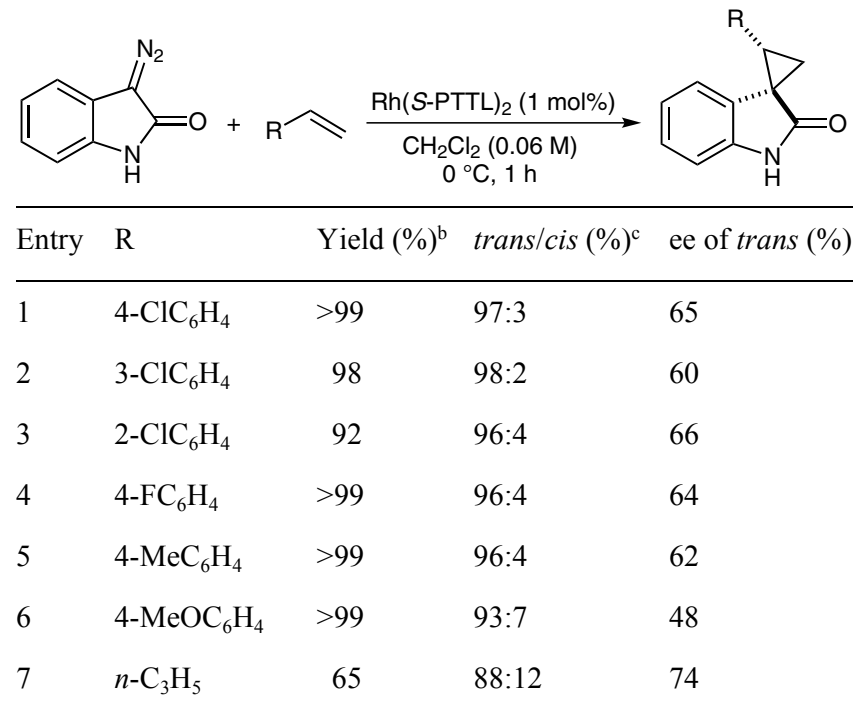

${ }^{a}$ Diazooxindole was added in one portion and stirred for $1 \mathrm{~h}$.

${ }^{\mathrm{b}}$ Combined yield of diastereomers.

${ }^{\mathrm{c}}$ Determined by crude NMR.

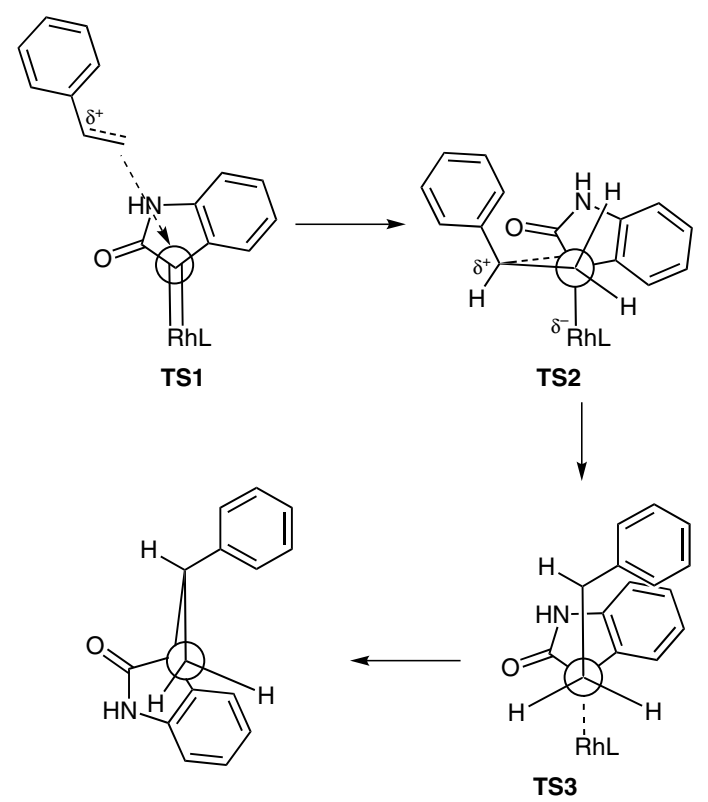

Scheme 2 Proposed mechanism for the trans-selective formation of spiro cyclopropyloxindole

struction of spiro-cyclopropyloxindoles was achieved successfully. This is the first report of an enantioselective reaction using diazooxindole. This novel method provides access to a variety of substituted cyclopropyloxindoles and will be useful in medicinal chemistry applications.

General Procedure for the Catalytic Asymmetric Cyclopropanation with Styrene and Diazooxindole (Table 2, Entry 6)

$\mathrm{Rh}_{2}(S \text {-PTTL })_{4}(0.0015 \mathrm{mmol}, 2.1 \mathrm{mg})$ was added to a two-necked round-bottom flask containing a magnetic stir bar under an Ar atmosphere, followed by addition of $\mathrm{CH}_{2} \mathrm{Cl}_{2}(0.5 \mathrm{~mL})$ to the flask. Styrene $(0.75 \mathrm{mmol})$ and diazooxindole $(0.15 \mathrm{mmol})$ in $\mathrm{CH}_{2} \mathrm{Cl}_{2}(2.0$
$\mathrm{mL}$ ) were added at $0{ }^{\circ} \mathrm{C}$. After stirring for $1 \mathrm{~h}$, the solvent was removed under reduced pressure, and the diastereomeric ratio was determined by crude ${ }^{1} \mathrm{H}$ NMR analysis. The resulting crude mixture was purified by silica gel column chromatography (hexane-EtOAc $=2: 1$ to $0: 1)$ to afford the cyclopropyloxindole. ${ }^{1} \mathrm{H}$ NMR $(500 \mathrm{MHz}$, $\left.\mathrm{CDCl}_{3}\right): \delta=9.39(\mathrm{br}, 1 \mathrm{H}), 7.30-7.19(\mathrm{~m}, 5 \mathrm{H}), 7.08(\mathrm{t}, J=7.7 \mathrm{~Hz}$, $1 \mathrm{H}), 6.96(\mathrm{~d}, J=7.5 \mathrm{~Hz}, 1 \mathrm{H}), 6.66(\mathrm{~m}, 1 \mathrm{H}), 5.95(\mathrm{~d}, J=7.5 \mathrm{~Hz}, 1$ $\mathrm{H}), 3.36(\mathrm{t}, J=8.6 \mathrm{~Hz}, 1 \mathrm{H}), 2.24-2.21(\mathrm{~m}, 1 \mathrm{H}), 2.04-2.02(\mathrm{~m}, 1 \mathrm{H})$; ${ }^{13} \mathrm{C} \mathrm{NMR}\left(125 \mathrm{MHz}, \mathrm{CDCl}_{3}\right): \delta=179.1,141.1,134.9,130.0,128.3$, 127.9, 127.4, 126.5, 121.4, 120.9, 109.7, 36.1, 33.7, 22.6; HRMS: $\mathrm{m} / z$ calcd for $\mathrm{C}_{16} \mathrm{H}_{12} \mathrm{NO}[\mathrm{M}-\mathrm{H}]: 234.0924$; found: 234.0928; IR (neat): $2922,1704,1619,1467,1218 \mathrm{~cm}^{-1} ;[\alpha]_{\mathrm{D}}^{25.3}=+104.3(c=$ $1.0, \mathrm{CHCl}_{3}, 98: 2 \mathrm{dr}, 66 \%$ ee); Enantiomeric excess was determined by HPLC with a Chiralpack AD-H column [hexane-2-PrOH $(70: 30), 1.0 \mathrm{~mL} / \mathrm{min}, 254 \mathrm{~nm}] ; t_{\mathrm{R}}$ (minor enantiomer) $=5.1 \mathrm{~min} ; t_{\mathrm{R}}$ $($ major enantiomer $)=6.3 \mathrm{~min}$.

\section{Acknowledgment}

This work was supported by a Grant-in-Aid for Scientific Research from the Ministry of Education, Culture, Sports, Science and Technology, Japan, and the Takeda Science Foundation.

Supporting Information for this article is available online at http://www.thieme-connect.com/ejournals/toc/synlett.

\section{References}

(1) Bindra, J. S. The Alkaloids; Vol. 14; Manske, R. H. F., Ed.; Academic Press: New York, 1973, 84.

(2) For reviews, see: (a) Galliford, C. V.; Scheidt, K. A. Angew. Chem. Int. Ed. 2007, 46, 8748; Angew. Chem. 2007, 119 , 8902 . (b) Marti, C.; Carreira, E. M. Eur. J. Org. Chem. 2003, 2209. (c) Trost, B. M.; Brennan, M. K. Synthesis 2009, 3003 .

(3) (a) Cui, C. B.; Kakeya, H.; Osada, H. Tetrahedron 1996, 52, 12651. (b) Cui, C. B.; Kakeya, H.; Osada, H. J. Antibiot. 1996, $49,832$.

(4) (a) Ding, K.; Lu, Y.; Nikolovska-Coleska, Z.; Qiu, S.; Ding, Y.; Gao, W.; Stuckey, J.; Krajewski, K.; Roller, P. P.; Tomita, Y.; Parrish, D. A.; Deschamps, J. R.; Wang, S. J. Am. Chem. Soc. 2005, 127, 10130. (b) Ding, K.; Lu, Y.; Nikolovska-Coleska, Z.; Wang, G.; Qiu, S.; Shangary, S.; Gao, W.; Qin, D.; Stuckey, J.; Krajewski, K.; Roller, P. P.; Wang, S. J. Med. Chem. 2006, 49, 3432. (c) Shangary, S.; Qin, D.; McEachern, D.; Liu, M.; Miller, R. S.; Qiu, S.; Nikolovska-Coleska, Z.; Ding, K.; Wang, G.; Chen, J.; Bernard, D.; Zhang, J.; Lu, Y.; Gu, Q.; Shah, R. B.; Pienta, K. J.; Ling, X.; Kang, S.; Guo, M.; Sun, Y.; Yang, D.; Wang, S. Proc. Natl. Acad. Sci. U.S.A. 2008, 105, 3933.

(5) (a) Stratmann, K.; Moore, R. E.; Bonjouklian, R.; Deeter, J. B.; Patterson, G. M. L.; Shaffer, S.; Smith, C. D.; Smitka, T. A. J. Am. Chem. Soc. 1994, 116, 9935. (b) Jimenez, J. I.; Huber, U.; Moore, R. E.; Patterson, G. M. L. J. Nat. Prod. 1999, 62, 569 .

(6) The total synthesis of welwitindolinone A isonitrile, see: (a) Baran, P. S.; Richter, J. M. J. Am. Chem. Soc. 2005, 127, 15394. (b) Reisman, S. E.; Ready, J. M.; Hasuoka, A.; Smith, C. J.; Wood, J. L. J. Am. Chem. Soc. 2006, 128, 1448

(7) (a) Jiang, T.; Kuhen, K. L.; Wolff, K.; Yin, H.; Bieza, K.; Caldwell, J.; Bursulaya, B.; Wu, T. Y.-H.; He, Y. Bioorg. Med. Chem. Lett. 2006, 16, 2105. (b) Jiang, T.; Kuhen, K. L.; Wolff, K.; Yin, H.; Bieza, K.; Caldwell, J.; Bursulaya, B.; Tuntland, T.; Zhang, K.; Karanewsky, D.; He, Y. Bioorg. Med. Chem. Lett. 2006, 16, 2109. 
(8) (a) Dandia, A.; Singh, R.; Saha, M.; Shivpuri, A. Pharmazie 2002, 57, 602. (b) Zhang, H.-P.; Kamano, Y.; Ichihara, Y.; Kizu, H.; Komiyama, K.; Itokawa, H.; Pettit, G. R. Tetrahedron 1995, 51, 5523. (c) Kamano, Y.; Zhang, H.-P.; Ichihara, Y.; Kizu, H.; Komiyama, K.; Pettit, G. R. Tetrahedron Lett. 1995, 36, 2783.

(9) Example of racemic synthesis of spiro-cyclopropane oxindoles, see: (a) Muthusamy, S.; Guanathan, C. Synlett 2003, 1599. (b) Chen, S.; Ma, J.; Wang, J. Tetrahedron Lett. 2008, 49, 6781; see also ref. 7 and 10 .

(10) Example of racemic synthesis of spiro-epoxyoxindoles, see: (a) Muthusamy, S.; Gunanathan, C.; Nethaji, M. Synlett 2004, 639. (b) Muthusamy, S.; Gunanathan, C.; Nethaji, M. J. Org. Chem. 2004, 69, 5631. (c) Schulz, V.; Davoust, M.; Lemari, M.; Lohier, J.-F.; Santos, J. S. O.; Metzner, P.; Brire, J.-F. Org. Lett. 2007, 9, 1745. (d) Muthusamy, S.; Karikalan, T.; Suresh, E. Tetrahedron Lett. 2011, 52, 1934.

(11) Awata, A.; Arai, T. Chem. Eur. J. 2012, 18, 8278.

(12) (a) Pesciaioli, F.; Righi, P.; Mazzanti, A.; Bartoli, G.; Bencivenni, G. Chem. Eur. J. 2011, 17, 2842. (b) Dou, X.; Lu, Y. Chem. Eur. J. 2012, 18, 8315. (c) Noole, A.; Sucman, N. S.; Kabeshov, M. A.; Kanger, T.; Macaev, F. Z.; Malkov, A. V. Chem. Eur. J. 2012, 18, 14929. For the catalytic asymmetric symthesis of spiro epoxyoxindole, see: (d) Palumbo, C.; Mazzeo, G.; Mazziotta, A.; Gambacorta, A.; Loreto, M. A.; Migliorini, A.; Superchi, S.; Tofani, D.; Gasperi, T. Org. Lett. 2011, 13, 6248.

(13) (a) Meyers, C.; Carreira, E. M. Angew. Chem. Int. Ed. 2003, 42, 694; Angew. Chem. 2003, 115, 718 . (b) Marti, C.; Carreira, E. M. J. Am. Chem. Soc. 2005, 127, 11505.

(14) For examples of $\mathrm{Cu}(\mathrm{I})$-catalyzed enantioselective cyclopropanation, see: (a) Fritschi, H.; Leutenegger, U.; Pfaltz, A. Helv. Chim. Acta 1988, 71, 1553. (b) Lowenthal, R. E.; Abiko, A.; Masamune, S. Tetrahedron Lett. 1990, 31, 6005. (c) Evans, D. A.; Woerpel, K. A.; Hinman, M. M.; Faul, M. M. J. Am. Chem. Soc. 1991, 113, 726. (d) Leutenegger, U.; Umbricht, G.; Fahrni, C.; von Matt, P.; Pfaltz, A. Tetrahedron 1992, 48, 2143. (e) Ito, K.; Katsuki, T. Synlett 1993, 638. (f) Lo, M. M.-C.; Fu, G. C. J. Am. Chem. Soc. 1998, 120, 10270.

(15) For examples of $\mathrm{Ru}(\mathrm{II})$-catalyzed enantioselective cyclopropanation, see: (a) Nishiyama, H.; Itoh, Y.; Matsumoto, H.; Park, S.-B.; Itoh, K. J. Am. Chem. Soc. 1994, 116, 2223. (b) Nishiyama, H.; Itoh, Y.; Sugawara, Y.; Matsumoto, H.; Aoki, K.; Itoh, K. Bull. Chem. Soc. Jpn. 1995, 68, 1247. (c) Miller, J. A.; Jin, W.; Nguyen, S. T. Angew. Chem. Int. Ed. 2002, 41, 2953. (d) Ito, J.; Ujiie, S.; Nishiyama, H. Chem. Eur. J. 2010, 16, 4986. (e) AbuElfotoh, A.-M.; Phomkeona, K.; Shibatomi, K.; Iwasa, S. Angew. Chem. Int. Ed. 2010, 49, 8439.

(16) For examples of Co(II)-catalyzed enantioselective cyclopropanation, see: (a) Nakamura, A.; Konishi, A.; Tatsuno, Y.; Otsuka, S. J. Am. Chem. Soc. 1978, 100, 3443. (b) Nakamura, A.; Konishi, A.; Tsujitani, R.; Kudo, M.; Otsuka, S. J. Am. Chem. Soc. 1978, 100, 3449. (c) Niimi, T.; Uchida, T.; Irie, R.; Katsuki, T. Adv. Synth. Catal. 2001, 343 , 79. (d) Ikeno, T.; Sato, M.; Sekino, H.; Nishizuka, A.; Yamada, T. Bull. Chem. Soc. Jpn. 2001, 74, 2139. (e) Ikeno, T.; Iwakura, I.; Yamada, T. J. Am. Chem. Soc. 2002, 124 , 15152. (f) Chen, Y.; Ruppel, J. V.; Zhang, X. P. J. Am. Chem. Soc. 2007, 129, 12074. (g) Chen, Y.; Zhang, X. P. J. Org. Chem. 2007, 72, 5931. (h) Shitama, H.; Katsuki, T. Chem. Eur. J. 2007, 13, 4849.
(17) For examples of $\mathrm{Rh}(\mathrm{II})$-catalyzed enantioselective cyclopropanation, see: (a) Doyle, M. P.; Brandes, B. D.; Kazala, A. P.; Pieters, R. J.; Jarstfer, M. B.; Watkins, L. M.; Eagle, C. T. Tetrahedron Lett. 1990, 31, 6613. (b) Doyle, M. P.; Winchester, W. R.; Hoorn, J. A. A.; Lynch, V.; Simonsen, S. H.; Ghosh, R. J. Am. Chem. Soc. 1993, 115, 9968. (c) Doyle, M. P.; Austin, R. E.; Bailey, A. S.; Dwyer, M. P.; Dyatkin, A. B.; Kalinin, A. V.; Kwan, M. M. Y.; Liras, S.; Oalmann, C. J.; Pieters, R. J.; Protopopova, M. N.; Raab, C. E.; Roos, G. H. P.; Zhou, Q.-L.; Martin, S. F. J. Am. Chem. Soc. 1995, 117, 5763. (d) Lou, Y.; Horikawa, M.; Kloster, R. A.; Hawryluk, N. A.; Corey, E. J. J. Am. Chem. Soc. 2004, 126, 8916; see also ref. 21.

(18) For selected examples of catalytic asymmetric cyclopropantion with aryl diazoacetates, see: (a) Doyle, M. P.; Zhou, Q.-L.; Charnsangavej, C.; Longoria, M. A.; McKervey, M. A.; García, C. F. Tetrahedron Lett. 1996, 37 , 4129. (b) Davies, H. M. L.; Bruzinski, P. R.; Fall, M. J. Tetrahedron Lett. 1996, 37, 4133. (c) Davies, H. M. L.; Nagashima, T.; Klino, J. L. III. Org. Lett. 2000, $2,823$. (d) Davies, H. M. L.; Townsend, R. J. J. Org. Chem. 2001, 66, 6595. (e) Davies, H. M. L.; Venkataramani, C. Org. Lett. 2003, 5, 1403. (f) Nowlan, D. T. III.; Gregg, T. M.; Davies, H. M. L.; Singleton, D. A. J. Am. Chem. Soc. 2003, 125, 15902. (g) Hedley, S. J.; Ventura, D. L.; Dominiak, P. M.; Nygren, C. L.; Davies, H. M. L. J. Org. Chem. 2006, 71, 5349. (h) Ventura, D. L.; Li, Z.; Coleman, M. G.; Davies, H. M. L. Tetrahedron 2009, 65, 3052. (i) Bonge, H. T.; Kaboli, M.; Hansen, T. Tetrahedron Lett. 2010, 51, 5375.

(19) (a) Watanabe, N.; Ogawa, T.; Ohtake, Y.; Ikegami, S.; Hashimoto, S. Synlett 1996, 85. (b) Saito, H.; Oishi, H.; Kitagaki, S.; Nakamura, S.; Anada, M.; Hashimoto, S. Org. Lett. 2002, 4, 3887. (c) Minami, K.; Saito, H.; Tsutsui, H.; Nambu, H.; Anada, M.; Hashimoto, S. Adv. Synth. Catal. 2005, 347, 1483. (d) Tsutsui, H.; Abe, T.; Nakamura, S.; Anada, M.; Hashimoto, S. Chem. Pharm. Bull. 2005, 53, 1366. (e) Takeda, K.; Oohara, T.; Anada, M.; Nambu, H.; Hashimoto, S. Angew. Chem. Int. Ed. 2010, 49, 6979. (f) DeAngelis, A.; Dmitrenko, O.; Yap, G. P. A.; Fox, J. M. J. Am. Chem. Soc. 2009, 131, 7230.

(20) (a) Davies, H. M. L.; Bruzinski, P. R.; Lake, D. H.; Kong, N.; Fall, M. J. J. Am. Chem. Soc. 1996, 118, 6897. (b) Hansen, J.; Autschbach, J.; Davies, H. M. L. J. Org. Chem. 2009, 74, 6555.

(21) (a) Watanabe, N.; Matsuda, H.; Kuribayashi, H.; Hashimoto, S. Heterocycles 1996, 42, 537. (b) Kitagaki, S.; Matsuda, H.; Watanabe, N.; Hashimoto, S. Synlett 1997, 1171. (c) Goto, T.; Takeda, K.; Anada, M.; Ando, K.; Hashimoto, S. Tetrahedron Lett. 2011, 52, 4200; and references cited therein.

(22) (a) Doyle, M. P.; Griffin, J. H.; Bagheri, V.; Dorow, R. L. Organometallics 1984, 3, 53. (b) Doyle, M. P.; Bagheri, V.; Wandless, T. J.; Harn, N. K.; Brinker, D. A.; Eagle, C. T.; Loh, K.-L. J. Am. Chem. Soc. 1990, 112, 1906. (c) O’Bannon, P. E.; Dailey, W. P. Tetrahedron 1990, 46, 7341

(23) A trans-directing ability of the amide group in the cyclopropanation, see: (a) Marcoux, D.; Charette, A. B. Angew. Chem. Int. Ed. 2008, 47, 10155. (b) Marcoux, D.; Goudreau, S. R.; Charette, A. B. J. Org. Chem. 2009, 74, 8939. 